\title{
An improved adaptive VMD method and its application in wear condition monitoring of main bearing
}

\author{
Jide Jia ${ }^{1}$, Gang Ren ${ }^{2}$ \\ ${ }^{1}$ The Higher Educational Key Laboratory for Flexible Manufacturing Equipment Integration of Fujian \\ Province, Xiamen Institute of Technology, Xiamen, China \\ ${ }^{1}$ State Key Laboratory for Manufacturing Systems Engineering, Xi' an Jiaotong University, Xian, China \\ ${ }^{2}$ Automobile NCO School, Army Military Transportation University, Bengbu, China \\ ${ }^{1}$ Corresponding author \\ E-mail: 1jjxyrengang@163.com, ${ }^{2} 18322018563 @ 163 . c o m$
}

Received 2 April 2021; received in revised form 12 April 2021; accepted 22 April 2021

DOI https://doi.org/10.21595/vp.2021.21987

Check for updates

Copyright $(2021$ Jide Jia, et al. This is an open access article distributed under the Creative Commons Attribution License, which permits unrestricted use, distribution, and reproduction in any medium, provided the original work is properly cited.

\begin{abstract}
The main bearing is a key component of internal combustion engine, which bear complex alternating load and easily break down due to wear. Therefore, monitoring the degradation of the main bearing is very important to avoid sudden mechanical failure. In order to solve the difficult problem of main bearing wear condition monitoring, an improved adaptive VMD is proposed. Firstly, different wear conditions of the main bearing are simulated to obtain vibration signals of the main bearing seat. Furthermore, on the basis of variational mode decomposition, the important parameter $K$ value of the algorithm is adaptive selected to optimize the decomposition of the vibration signal to be processed. Finally, the wear characteristics of the main bearing are extracted and the wear state of the main bearing is distinguished according to the threshold value by comparing the energy changes of each inherent modal component under different wear conditions. Through the analysis of the simulation signal and the actual vibration signal, the results show that this method can effectively monitor the state of the main bearing.
\end{abstract}

Keywords: internal combustion engine; main bearing; condition monitoring; improved adaptive VMD.

\section{Introduction}

As the key component of internal combustion engine, the main bearing is sliding bearing, which has the advantages of strong bearing capacity, stable operation and low noise. The main bearing bears complex alternating load, the working environment is very complex and the working condition is very bad. Wear failure often occurs, leading to crankshaft bearing burning and crankshaft fracture. Therefore, it is very important to carry out on-line condition monitoring of main bearing [1].

When internal combustion engine is working, there are both reciprocating motion of piston and rotary motion of crankshaft, which lead to the vibration signal of internal combustion engine has the characteristics of multi-component, non-stationary and non-linear. It includes the impact caused by the collision between crankshaft journal and crankshaft bearing, connecting rod journal and connecting rod bearing, piston and cylinder wall, intake and exhaust valve and valve seat, and various signal components caused by the friction between journal and bearing.

How to extract wear fault features of main bearing from complex vibration signals of internal combustion engine is the key of on-line condition monitoring of main bearing. Wavelet transform can be used to analyze complex signal with flexible window (window function size unchanged but shape changed). In reference [2], the energy singularity of vibration signal's modulus maximum point is detected by using dyadic discrete wavelet transform, and the fault diagnosis of crankshaft bearing is realized. However, the time-frequency resolution of wavelet is limited by Heisenberg's uncertainty principle and cannot be optimized at the same time. The result of signal analysis is greatly influenced by the choice of wavelet basis. In reference [3], combined with empirical mode decomposition (EMD) and Gabor transform, the frequency band energy 
characteristics reflecting the wear state of crankshaft bearings are successfully extracted. In reference [4], integrated empirical mode decomposition (EEMD) is introduced and combined with fuzzy $c$-means clustering to realize the accurate identification of crankshaft bearing fault state. However, EMD lacks strict mathematical basis, has poor anti noise ability and mode aliasing. EEMD is an improved analysis method based on EMD, but it still cannot avoid the endpoint effect, large amount of calculation and poor denoising ability.

Variational mode decomposition (VMD) is a new adaptive time-frequency analysis theory [5]. VMD realizes the frequency domain decomposition of the signal and the effective separation of the modal components in a completely non recursive way. It has the advantages of strong decomposition ability, good denoising performance and fast algorithm speed. The decomposition effect of VMD is affected by the $K$ value of the decomposed signal component, that is, too much or insufficient decomposition will affect the accuracy of the analysis results. When $k$ is too large, there will be over decomposition, and the center frequencies of each mode will gather or even overlap. If $K$ is too small and under decomposed, some modes will be divided into adjacent modes or even discarded. Therefore, how to choose the appropriate $K$ value before decomposition is the key to the wide application of VMD.

At present, the most common method to select $k$ value is to observe the center frequency of IMF decomposed by VMD. When the frequency distance between adjacent IMF centers is close, it is considered that there is over decomposition and $K$ value is determined [6]. It is troublesome to determine $K$ value by observation, which cannot meet the needs of on-line condition monitoring of main bearing. In reference [7], genetic algorithm is used to determine the value of modal number $k$ and realize the adaptive determination of $K$ value. However, this method takes a long time to calculate and is not suitable for on-line monitoring. The $k$-value problem needs to be further optimized.

In this paper, an improved adaptive VMD is proposed. Firstly, the first-order difference method is used to automatically obtain the distance between the center frequencies of the decomposed IMF, and the minimum difference value is compared to determine the optimal $K$ value of VMD decomposition. This is a new method to determine $K$ value, which is helpful to realize real-time monitoring. The proposed method is verified by simulation signal and vibration signal analysis of main bearing seat. The results show that the method can eliminate the strong noise interference, extract the transient impact characteristics of diesel engine combustion cycle vibration, and effectively identify the misfire fault of diesel engine.

\section{Fault diagnosis principle of improved adaptive VMD}

\subsection{Variational mode decomposition}

According to the Reference [8], we get the complete algorithm for VMD, summarized in algorithm 1 .

Algorithm 1: Complete optimization of VMD:

Initialize $\left\{\hat{u}_{k}^{1}\right\},\left\{\widehat{\omega}_{k}^{1}\right\},\left\{\hat{\lambda}_{k}^{1}\right\}, n \leftarrow 0$.

Repeat $n \leftarrow 0$.

For $k=1: K$ do.

Update $\hat{u}_{k}^{n+1}$ for all $w>=0$ :

$\hat{u}_{k}^{n+1}(\omega) \leftarrow \frac{f(\omega)-\sum_{i} \hat{u}_{k}(\omega)+\frac{\hat{\lambda}(\omega)}{2}}{1+2 \alpha\left(\omega \omega_{k}\right)^{2}}$.

Update $w_{k}$ : 
$\omega_{k}^{n+1}(\omega) \leftarrow \frac{\int_{0}^{\infty} \omega\left|\hat{u}_{k}(\omega)\right|^{2} d \omega}{\int_{0}^{\infty}\left|\hat{u}_{k}(\omega)\right|^{2} d \omega}$

end for.

Dual ascent for all $w>=0$ :

$\hat{\lambda}^{n+1}(\omega) \leftarrow \hat{\lambda}^{n}(\omega)+\beta\left[\hat{f}(\omega) \sum_{k=1}^{K} \hat{u}_{k}^{n+1}(\omega)\right]$

until convergence:

$\frac{\sum_{k=1}^{K}\left\|\hat{u}_{k}^{n+1} \hat{u}_{k}^{n}\right\|_{2}^{2}}{\left\|\hat{u}_{k}^{n}\right\|_{2}^{2}}<2$.

The reconfiguration signal can be expressed as:

$\hat{f}(t)=\sum_{k=1}^{K} \hat{u}_{k}$

\subsection{Improved adaptive VMD}

The fault diagnosis of main bearing based on adaptive VMD is based on optimizing the $K$ value of VMD. The specific steps are as follows:

1) The vibration signal on the main bearing seat is collected by the acceleration sensor.

2) The VMD method is used to decompose the vibration signal. The candidate $K$ values are traversed in the preset range, and the center frequencies of IMF components under different candidate $K$ values are calculated. The center frequency matrix CF of IMF component is obtained:

$C F=\left[\begin{array}{lllll}\omega_{11} & \omega_{12} & & & \\ \omega_{21} & \omega_{22} & \omega_{23} & & \\ & & & \ddots & \\ \omega_{i 1} & \omega_{i 2} & \omega_{i 3} & \ldots & \omega_{i K}\end{array}\right]$.

3) The first-order difference matrix DF is obtained by calculating the first-order difference of the IMF central frequency matrix CF:

$d F=\left[\begin{array}{cccc}d \omega_{11} & \omega_{12} & & \\ d \omega_{21} & d \omega_{22} & & \\ & & \ddots & \\ d \omega_{i 1} & d \omega_{i 2} & \ldots & d \omega_{i K-1}\end{array}\right]$.

4) The last value in each row of the first-order difference matrix of IMF center frequency is selected to establish a one-dimensional vector $D$ :

$D=\left[\begin{array}{llll}d \omega_{11} & d \omega_{22} & d \omega_{3_{3}} \ldots d \omega_{i K-1}\end{array}\right]$.

5) Find the minimum value min $(d)$ of the vector, and the number of IMF decomposition corresponding to the minimum value minus 1 is the optimal number of solutions.

6) The vibration signal is decomposed by the optimal number $\mathrm{k}$ of IMF, and the energy of $K$ IMF is calculated: 
$E_{i}=\sum_{j=1}^{n}\left[u_{i j}(t)\right]^{2}$

where $u_{i j}$ is the ith IMF and $E$ is the energy of the $i$ th IMF.

\section{Experimental results}

\subsection{Experiment condition}

The test was carried out on Cummins 6bt5.9 diesel engine. Different fit clearances were set in the fourth crankshaft bearing to simulate different wear states. In practice, only single bearing wear is rare. Therefore, in order to meet the actual law, when setting bearing failure, change the matching clearance of two adjacent bearings. See Table 1 for specific settings. The vibration acceleration sensor is fixed on the fourth crankshaft bearing seat to measure the vibration signal of the main bearing seat when the crankshaft bearing has different fit clearance. The sampling frequency is $20 \mathrm{kHz}$ and the length of each data sample is 80000 . During the test, the diesel engine ran at no-load and the speed was kept in the range of $1850 \mathrm{r} / \mathrm{min}-1950 \mathrm{r} / \mathrm{min}$. The signal line of vibration acceleration sensor is led out through the joint of diesel engine block and oil pan. The arrangement of measuring points is shown in Fig. 1.

Table 1. diesel engine main bearing different wear state settings

\begin{tabular}{|c|c|c|c|c|c|}
\hline \multirow{2}{*}{ Fault name } & \multirow{2}{*}{ Set position } & \multicolumn{4}{|c|}{ Failure level (mm) } \\
\cline { 3 - 6 } & & Normal & Slight & Moderate & Serious \\
\hline \multirow{3}{*}{ Main bearing wear } & Third crankshaft bearing and journal & $0.08-0.1$ & $0.16-0.18$ & $0.24-0.26$ & $0.38-0.4$ \\
\cline { 2 - 6 } & Fourth crankshaft bearing and journal & $0.08-0.1$ & $0.24-0.26$ & $0.38-0.4$ & $0.53-0.55$ \\
\cline { 2 - 6 } & Fifth crankshaft bearing and journal & $0.08-0.1$ & $0.16-0.18$ & $0.24-0.26$ & $0.24-0.26$ \\
\hline
\end{tabular}

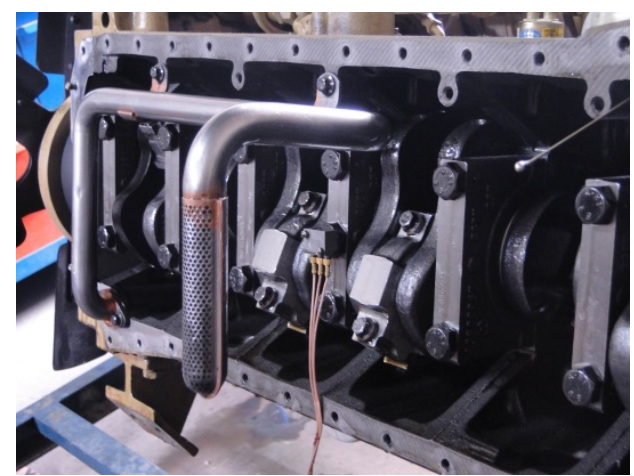

Fig. 1. Installation position of vibration sensor

\subsection{Experimental data processing}

The signal waveform of measuring points on the main bearing seat is shown in Fig. 2. It can be seen from the figure that the vibration signal of the measuring point is very complex, including multiple impact components. Although the diesel engine keeps running at about $1850 \mathrm{r} / \mathrm{min}-1950 \mathrm{r} / \mathrm{min}$, the vibration energy presents a wide-band distribution in the frequency domain, which indicates that the working process of the diesel engine is non-stationary and time-varying.

The vibration signals of four main bearing wear states are decomposed by VMD, and the $K$ value of VMD is 3, as shown in Fig. 3.

VMD is used to decompose vibration signals of different wear states and extract IMF energy. The energy of each IMF under different wear conditions is shown in Table 2. It can be seen from 
Table 2 that the change of frequency band energy can accurately reflect different wear states of crankshaft bearings. By monitoring the energy change of this frequency band, the early crankshaft bearing wear fault diagnosis can be realized without disassembly.

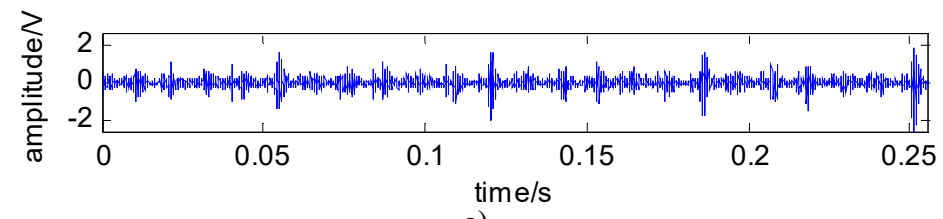

a)

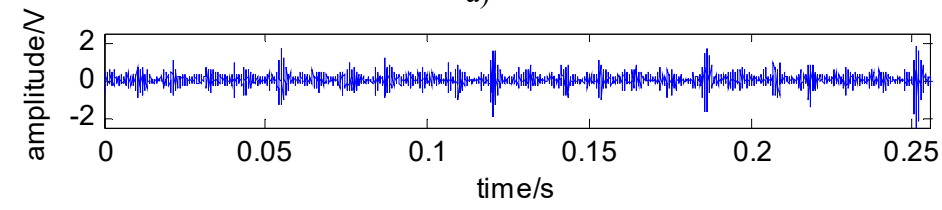

b)

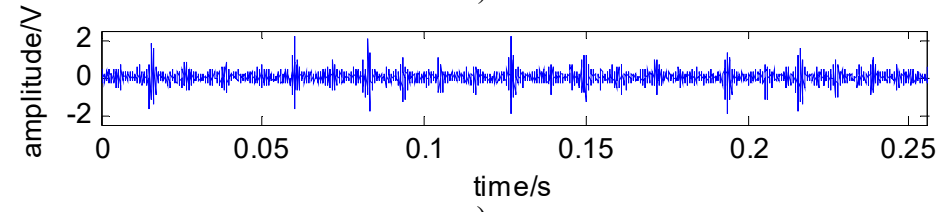

c)

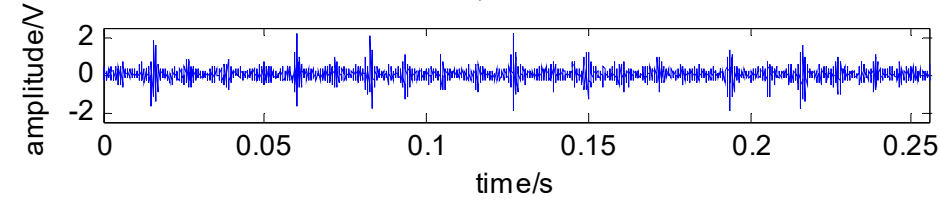

d)

Fig. 2. Vibration signal waveform of main bearing: a) normal, b) mild, c) moderate, d) severe

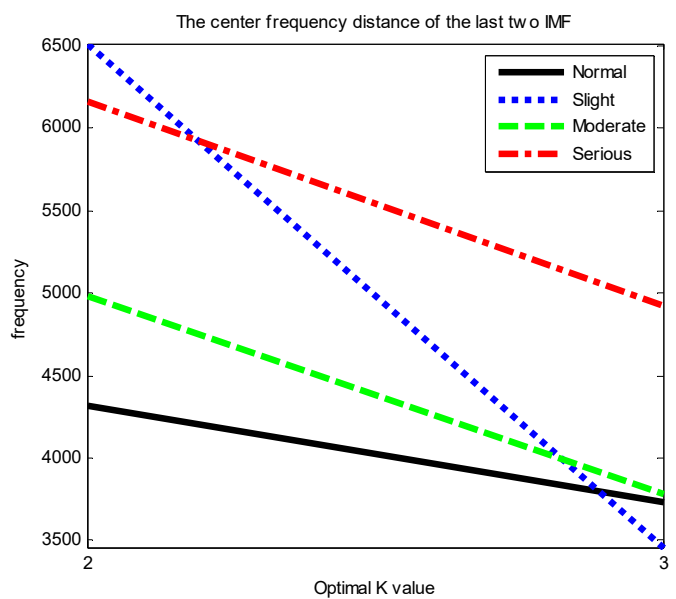

Fig. 3. Optimum $K$ value of wear signal of main bearing

Table 2. Energy of each modal component in different wear states

\begin{tabular}{|c|c|c|l|}
\hline Wear condition & IMF1 & IMF2 & \multicolumn{1}{|c|}{ IMF3 } \\
\hline Normal wear & 1.9446 & 1.5021 & 12.7051 \\
\hline Slight wear & 2.2485 & 1.6635 & 37.1284 \\
\hline Medium wear & 4.2006 & 1.6017 & 6.3499 \\
\hline Severe wear & 9.9689 & 2.4762 & 51.4680 \\
\hline
\end{tabular}




\section{Conclusions}

In this paper, a new improved adaptive VMD is proposed for the wear failure of crankshaft bearing of diesel engine. Firstly, the first-order difference method is used to automatically obtain the distance between the center frequencies of the decomposed IMF, and the minimum difference value is compared to determine the optimal $K$ value of VMD decomposition. The proposed method realizes the adaptive selection of $K$ value, which makes it possible to monitor the wear state of main bearing online.

The experimental results show that the proposed method can be used for feature extraction and fault diagnosis of diesel engine main bearing wear fault signal, which provides theoretical basis for crankshaft bearing wear fault diagnosis mechanism. Based on the vibration propagation model between the fault excitation source and the measuring point of the engine block, the fault characteristic frequency band $256 \mathrm{~Hz}-1024 \mathrm{~Hz}$ is extracted from the engine block signal. The energy change of the frequency band can accurately reflect the different wear states of crankshaft bearing. By monitoring the energy change of the frequency band, the early wear fault diagnosis of crankshaft bearing can be realized without disassembly.

\section{References}

[1] Jia J. D., Zang D., Mei J. M., et al. Signal Processing Method for Vehicle Fault Diagnosis. Chemical Industry Press, Beijing, 2015.

[2] Xiao Y. K., Qiao L., Zhang L. L., et al. A Study on engine fault diagnosis method based on wavelet singularity detection. Automotive Engineering, Vol. 36, Issue 11, 2014, p. 1405-1409.

[3] Zang Y. P., Zhang D. J., Wang W. Z. Fault diagnosis of engine abnormal sound based on wavelet transform technique. Journal of Mechanical Engineering, Vol. 45, Issue 6, 2009, p. 239-245.

[4] Zhang L. L., Liao H. Y., Cao Y. J., et al. Diagnosis on crankshaft bearing fault based on EEMD and fuzzy C mean clustering arithmetic. Transactions of CSICE, Vol. 29, Issue 4, 2011, p. 332-336.

[5] Zosso D., Dragomiretskiy K. Variational mode decomposition. IEEE Transactions on Signal Processing, Vol. 62, Issue 3, 2014, p. 531-544.

[6] Jia J. D., Ren G., Mei J. M. Misfire fault diagnosis of diesel engine based on VMD and XWT. Vibroengineering Procedia, Vol. 24, 2019, p. 17-22.

[7] Gang R., Jia J., Mei J., et al. An improved variational mode decomposition method and its application in diesel engine fault diagnosis. Journal of Vibroengineering, Vol. 20, Issue 6, 2018, p. 2363-2378.

[8] Dragomiretskiy K., Zosso D. Variational mode decomposition. IEEE Transactions on Signal Processing, Vol. 62, Issue 3, 2013, p. 531-544. 